\title{
Asymptotic behavior of the Coulomb three-body scattered wave
}

\author{
A. S. Kadyrov, ${ }^{1}$ A. M. Mukhamedzhanov, ${ }^{2}$ A. T. Stelbovics, ${ }^{1}$ I. Bray, ${ }^{1}$ and F. Pirlepesov ${ }^{2}$ \\ ${ }^{1}$ Centre for Atomic, Molecular and Surface Physics, School of Mathematical and Physical Sciences, Murdoch University, \\ Perth 6150, Australia \\ ${ }^{2}$ Cyclotron Institute, Texas A\&M University, College Station, Texas 77843, USA
}

(Received 11 April 2003; published 13 August 2003)

\begin{abstract}
Asymptotic forms of the scattered wave for a system of three arbitrary charged particles valid in all domains relevant to breakup are derived. The derivations are based on the relationship between the total wave function of a breakup process in a three-body system with Coulomb interactions and the wave function of the process of scattering of all three particles of the system in the continuum. The results are free from amplitude-phase ambiguity problems associated with previously known forms. A similar technique is used to obtain asymptotic forms of the three-body Coulomb Green's function.
\end{abstract}

DOI: 10.1103/PhysRevA.68.022703

PACS number(s): 03.65.Nk, 34.10.+x, 21.45.+v

\section{INTRODUCTION}

With the progress in high-performance computing, the direct numerical solution of the Schrödinger equation (see Refs. [1-5] and references therein) has emerged as a powerful method to analyze the scattering processes with three charged particles. Such methods, in principle, require the knowledge of the asymptotic behavior of the scattered wave function in all asymptotic regions of the configuration space. This is because asymptotic wave functions are used directly as boundary conditions in solving the differential equation mentioned above, or for extracting the scattering amplitudes from integral expressions involving the full scattering wave function. Below the three-body breakup threshold when only two-cluster channels are open, there is no difficulty with the application of the aforementioned approaches in combination with some additional, but reasonable, approximation schemes. However, we emphasize that attempts to follow exact algorithms have thus far limited these approaches to essentially model problems [6-9].

Above the breakup threshold, the situation is different. Here, a required unique solution is obtained by imposing the boundary conditions in the regions where all three particles are asymptotically free. The most studied system is that of electron-hydrogen scattering. Lack of knowledge of the complete asymptotic behavior of the scattered wave function has led to different approaches, aimed at "avoiding" the true three-body asymptotic state, including exterior complex scaling (ECS) [1], convergent close coupling (CCC) [10], $T$-matrix [2], and $R$-matrix [3,11] methods. These methods provide an accurate three-body scattering wave function in an "internal" region in coordinate space and the ionization amplitude is extracted by matching to ionization boundary conditions in the asymptotic region. In each method, the extraction process relies on approximate ionization boundary conditions. For example, in the CCC method, the ionization flux is initially obtained by discretizing the target continuum. The ionization amplitude is then constructed by means of a renormalization of the square-integrable positive-energy target states with the true target continuum [12]. Implicit in this approach is the representation of the three-body continuum states as a product of plane and Coulomb waves without electron-electron correlation. In ECS [1], T-matrix [2], and $R$-matrix with $T$-matrix [11] methods, an integral representation of the ionization amplitude is used but again the threebody continuum states are approximated, this time by a product of two fixed-charge Coulomb waves for the two free electrons. This yields an ionization amplitude with divergent phase as a function of matching radius although the magnitude of the amplitude converges. Thus, due to the necessity to eventually calculate the flux at infinity, none of these methods can really avoid the asymptotic form of the scattered wave, rather they approximate it.

Despite the success of these practical approaches in providing accurate cross sections, formal theory of breakup with charged particles remains incomplete. The formal theory given over 30 years ago [13-15] is still considered state of the art. Within the framework of this theory, there is no, and cannot be, exact algorithm, e.g., similar to Poet's [7] below the breakup threshold, for solution of the Schrödinger equation with correct boundary conditions above the breakup threshold. The first and the only attempt to solve the Schrödinger equation for electron-impact ionization of hydrogen by directly matching with exact ionization boundary conditions is limited to the $S$-wave model [16]. Though an asymptotic form of the scattered wave for electron-impact ionization of hydrogen for the case when all interparticle distances are large was obtained by Peterkop $[17,18]$ four decades ago, it has not been successfully implemented in the aforementioned approaches. One reason is that the direct numerical solution of the Schrödinger equation for the full hydrogen-ionization problem requires partial-wave analysis of the asymptotic wave function and a suitable partial-wave decomposition of the Peterkop wave function does not exist. The problem with the partial-wave decomposition is that Peterkop's asymptotic wave function is invalid when two electrons are close to each other. Thus, for full-scale numerical calculations, a representation of the wave function, describing ionization in this region as well, is necessary.

Peterkop used six-dimensional hyperspherical coordinates which effectively transform the Schrödinger equation describing the development of the system into the HamiltonJacobi-type equation as the asymptotic motion of the particles becomes classical. For this reason, the Peterkop 
asymptotic wave suffers from an amplitude-phase ambiguity problem, since some part of the hyperspherical ionization amplitude can be moved to the phase factor and the resulting wave function is still a solution to the original HamiltonJacobi equation [15]. Accordingly, the remaining amplitude can equally be called an ionization amplitude. Thus, generally speaking, the hyperspherical approach is not capable of uniquely identifying the ionization amplitude. This amplitude-phase ambiguity has caused problems in the formal theory of breakup at a very fundamental level. As a result, the present formal scattering theory cannot provide full information about the breakup amplitude.

Finally, full knowledge of the asymptotic behavior of the scattered wave forms the basis for the Kohn variational approach to breakup scattering [19-21]. Due to the absence of the asymptotic wave function for breakup scattering of three charged particles, validity of the variational approach to such processes was shown [22] only in the region where distances between all particles are large. For the case of protondeuteron breakup, for example, validity of the variational principle when in the final state all three particles are in the continuum but the neutron is close to one of the protons and the other proton is far away is yet to be proven. Therefore, for the recent Kohn variational proton-deuteron scattering calculations [21] to be extended to calculations of the deuteron breakup amplitude, an unambiguous asymptotic form of the total scattered wave is necessary. In this case, it is expected that the proton, which is far away, distorts the relative motion of the other proton and neutron due to the longrange Coulomb interaction between the protons. Thus, the knowledge of the asymptotic behavior of the three-body scattered wave in all regions of the configuration space is crucial in calculations of atomic and nuclear breakup processes.

In the preceding paper [23], a relationship between the total wave function describing ionization in the electronhydrogen system and the one representing scattering of three particles of the system in the continuum was established. On the basis of this relationship, forms of the scattered wave for ionization valid in all asymptotic domains relevant to ionization were obtained and the amplitude-phase ambiguity of the Peterkop wave function was resolved. This removed the above-mentioned problems in practical calculations and made the correct extraction of observables possible. In the present paper, these results are generalized to an arbitrary system of three charged particles and allowance is made for inclusion of short-range potentials as well as their Coulomb tails. We also go into some more detail of the derivation which was beyond the limits of the preceding brief report. In addition, we use a similar technique to obtain asymptotic forms of the three-body Coulomb Green's function. The latter are important in the formulation of the three-body problem $[24,25]$. Asymptotic forms of the three-particle Green's function also play a central role, for instance, when calculating the optical potentials [26-28] and doing the nonperturbative calculations of dynamical dipole polarization terms [29].

The paper is set out as follows. In Sec. II, we give a relationship between the total wave function of a breakup process in two-cluster collisions taking place in an arbitrary Coulomb three-body system and the wave function of the process of scattering of all three particles of the system in the continuum. Calculations of asymptotic forms of the scattered wave based on this relationship are presented in Sec. III. Asymptotic forms of the three-body Green's function are given in Sec. IV. Finally, in Sec. V, we summarize the results of the present work and discuss their possible applications.

\section{RELATIONSHIP BETWEEN $2 \rightarrow 3$ AND $3 \rightarrow 3$ PROCESSES IN A COULOMB THREE-BODY SYSTEM}

Let us consider a system of three particles of mass $m_{\alpha}$ and charge $z_{\alpha}, \alpha=1,2,3$. We use a system of Jacobi variables: $\boldsymbol{r}_{\alpha}$ is the relative coordinate, and $\boldsymbol{k}_{\alpha}$ is the relative momentum, between particles $\beta$ and $\gamma ; \boldsymbol{\rho}_{\alpha}$ is the relative coordinate of the center of mass of the pair $(\beta, \gamma)$ and particle $\alpha$, with $\boldsymbol{q}_{\alpha}$ being the canonically conjugate relative momentum. The corresponding reduced masses are denoted by $\mu_{\alpha}$ $=m_{\beta} m_{\gamma} /\left(m_{\beta}+m_{\gamma}\right) \quad$ and $\quad M_{\alpha}=m_{\alpha}\left(m_{\beta}+m_{\gamma}\right) /\left(m_{\alpha}+m_{\beta}\right.$ $\left.+m_{\gamma}\right)$. Here and throughout this paper, $\beta, \gamma=1,2,3, \alpha \neq \beta$ $\neq \gamma$. We use units such that $\hbar=c=1$.

For further reference, we note that

$$
\boldsymbol{r}_{\beta}=-\frac{\mu_{\alpha}}{m_{\gamma}} \boldsymbol{r}_{\alpha}-\boldsymbol{\epsilon}_{\beta \alpha} \boldsymbol{\rho}_{\alpha}, \quad \boldsymbol{\rho}_{\beta}=\epsilon_{\beta \alpha} \frac{\mu_{\beta}}{M_{\alpha}} \boldsymbol{r}_{\alpha}-\frac{\mu_{\beta}}{m_{\gamma}} \boldsymbol{\rho}_{\alpha}
$$

and

$$
\boldsymbol{k}_{\beta}=-\frac{\mu_{\beta}}{m_{\gamma}} \boldsymbol{k}_{\alpha}-\epsilon_{\beta \alpha} \frac{\mu_{\alpha}}{M_{\beta}} \boldsymbol{q}_{\alpha}, \quad \boldsymbol{q}_{\beta}=\boldsymbol{\epsilon}_{\beta \alpha} \boldsymbol{k}_{\alpha}-\frac{\mu_{\alpha}}{m_{\gamma}} \boldsymbol{q}_{\alpha},
$$

where $\epsilon_{\beta \alpha}=-\epsilon_{\alpha \beta}$ is the antisymmetric symbol, with $\epsilon_{\beta \alpha}$ $=1$ for $(\beta \alpha)$ being a cyclic permutation of $(1,2,3)$, and $\epsilon_{\alpha \alpha}=0$.

Consider the scattering of particle $\alpha$ with incident momentum $\boldsymbol{q}_{i}$ off a bound pair $(\beta, \gamma)$ in initial state $\phi_{n_{i}}\left(\boldsymbol{r}_{\alpha}\right)$ of energy $E_{n_{i}}$. Assume that the energy of the projectile $q_{i}^{2} / 2 M_{\alpha}$ is enough to break up the target. The total three-body wave function describing this process satisfies the Schrödinger equation

$$
(E-H) \Phi_{\boldsymbol{k}_{\alpha}, \boldsymbol{q}_{\alpha}}^{+}\left(\boldsymbol{r}_{\alpha}, \boldsymbol{\rho}_{\alpha}\right)=0,
$$

where $\quad H=-\Delta_{r_{\alpha}} / 2 \mu_{\alpha}-\Delta_{\boldsymbol{\rho}_{\alpha}} / 2 M_{\alpha}+V_{\alpha}\left(\boldsymbol{r}_{\alpha}\right)+V_{\beta}\left(\boldsymbol{r}_{\beta}\right)$ $+V_{\gamma}\left(\boldsymbol{r}_{\gamma}\right)$ is the three-body Hamiltonian and $E=E_{n_{i}}$ $+q_{i}^{2} / 2 M_{\alpha}=k_{\alpha}^{2} / 2 \mu_{\alpha}+q_{\alpha}^{2} / 2 M_{\alpha}$ is the total energy of the system. Here

$$
V_{\alpha}\left(\boldsymbol{r}_{\alpha}\right)=V_{\alpha}^{C}\left(\boldsymbol{r}_{\alpha}\right)+V_{\alpha}^{S}\left(\boldsymbol{r}_{\alpha}\right), \quad V_{\alpha}^{C}\left(\boldsymbol{r}_{\alpha}\right)=\frac{z_{\beta} z_{\gamma}}{r_{\alpha}},
$$

where $V_{\alpha}^{C}\left(V_{\alpha}^{S}\right)$ is the Coulomb (short-range) interaction between particles $\beta$ and $\gamma$.

The wave function $\Phi^{+}$consists of the incoming initialchannel wave $\Phi^{(i)}$ and outgoing scattered wave $\Phi^{(\mathrm{sc})+}$ :

$$
\Phi_{\boldsymbol{k}_{\alpha}, \boldsymbol{q}_{\alpha}}^{+}\left(\boldsymbol{r}_{\alpha}, \boldsymbol{\rho}_{\alpha}\right)=\Phi_{n_{i}, \boldsymbol{q}_{i}}^{(i)}\left(\boldsymbol{r}_{\alpha}, \boldsymbol{\rho}_{\alpha}\right)+\Phi_{\boldsymbol{k}_{\alpha}, \boldsymbol{q}_{\alpha}}^{(\mathrm{scc}+}\left(\boldsymbol{r}_{\alpha}, \boldsymbol{\rho}_{\alpha}\right),
$$


where $\Phi^{(i)}$ is separable and given by

$$
\Phi_{n_{i}, \boldsymbol{q}_{i}}^{(i)}\left(\boldsymbol{r}_{\alpha}, \boldsymbol{\rho}_{\alpha}\right)=\chi_{\boldsymbol{q}_{i}}\left(\boldsymbol{\rho}_{\alpha}\right) \phi_{n_{i}}\left(\boldsymbol{r}_{\alpha}\right) .
$$

The initial-channel two-body bound state is determined from

$$
\left(\frac{1}{2 \mu_{\alpha}} \Delta_{r_{\alpha}}-V_{\alpha}\left(\boldsymbol{r}_{\alpha}\right)+E_{n_{i}}\right) \phi_{n_{i}}\left(\boldsymbol{r}_{\alpha}\right)=0 .
$$

The wave function describing the relative motion of two clusters satisfies the following equation:

$$
\left(\frac{1}{2 M_{\alpha}} \Delta_{\boldsymbol{\rho}_{\alpha}}-U_{\alpha}\left(\boldsymbol{\rho}_{\alpha}\right)+\frac{q_{i}^{2}}{2 M_{\alpha}}\right) \chi_{q_{i}}\left(\boldsymbol{\rho}_{\alpha}\right)=0,
$$

where $U_{\alpha}\left(\boldsymbol{\rho}_{\alpha}\right)$ describes the interaction of the incident particle $\alpha$ with the c.m. of the bound subsystem $(\beta, \gamma)$ and is written as

$$
U_{\alpha}\left(\boldsymbol{\rho}_{\alpha}\right)=V_{\beta}\left(\boldsymbol{\rho}_{\alpha}\right)+V_{\gamma}\left(\boldsymbol{\rho}_{\alpha}\right) .
$$

Taking into account Eqs. (7) and (8), Eq. (3) can be rewritten as

$$
(E-H) \Phi_{\boldsymbol{k}_{\alpha}, \boldsymbol{q}_{\alpha}}^{(\mathrm{sc})+}\left(\boldsymbol{r}_{\alpha}, \boldsymbol{\rho}_{\alpha}\right)=\bar{V}_{\alpha} \Phi_{n_{i}, \boldsymbol{q}_{i}}^{(i)}\left(\boldsymbol{r}_{\alpha}, \boldsymbol{\rho}_{\alpha}\right),
$$

where

$$
\bar{V}_{\alpha}=V_{\beta}\left(\boldsymbol{r}_{\beta}\right)+V_{\gamma}\left(\boldsymbol{r}_{\gamma}\right)-U_{\alpha}\left(\boldsymbol{\rho}_{\alpha}\right),
$$

with $V_{\beta}\left(\boldsymbol{r}_{\beta}\right)+V_{\gamma}\left(\boldsymbol{r}_{\gamma}\right)$ term being the interaction of the projectile particle with the individual target particles. Then, applying the three-body Green's function $G^{+}=(E-H$ $+i 0)^{-1}$ to both sides of Eq. (10), we get

$$
\begin{aligned}
& \Phi_{k_{\alpha}, \boldsymbol{q}_{\alpha}}^{(\mathrm{sc})+}\left(\boldsymbol{r}_{\alpha}, \boldsymbol{\rho}_{\alpha}\right) \\
& =\int d \boldsymbol{r}_{\alpha}^{\prime} d \boldsymbol{\rho}_{\alpha}^{\prime} G^{+}\left(\boldsymbol{r}_{\alpha}, \boldsymbol{\rho}_{\alpha} ; \boldsymbol{r}_{\alpha}^{\prime}, \boldsymbol{\rho}_{\alpha}^{\prime} ; E+i 0\right) \\
& \quad \times \bar{V}_{\alpha} \Phi_{n_{i}, q_{i}}^{(i)}\left(\boldsymbol{r}_{\alpha}^{\prime}, \boldsymbol{\rho}_{\alpha}^{\prime}\right) .
\end{aligned}
$$

Next we apply a spectral decomposition for the Green's function. To this end, we consider another scattering process within the same three-body system, but one where in the initial channel all three particles are in the continuum (socalled $3 \rightarrow 3$ scattering as opposed to $2 \rightarrow 3$ breakup scattering in two-cluster collisions). We take the boundary condition for the wave function $\Psi^{-}$describing this process in the form of a Coulomb-distorted three-body plane wave and incoming scattered wave. This wave function, of course, is also an eigenstate of the same Hamiltonian $H$, i.e., ( $E$ $-H) \Psi_{\boldsymbol{k}_{\alpha}, \boldsymbol{q}_{\alpha}}^{-}\left(\boldsymbol{r}_{\alpha}, \boldsymbol{\rho}_{\alpha}\right)=0$. Therefore, it is well suited to our purpose. As it will become clear below, the reason for choosing this form of the total wave function as the basis for decomposition rather than $\Psi^{+}$, which consists of Coulombdistorted three-body plane wave and outgoing scattered wave, is twofold. First, using $\Psi^{+}$would eventually lead to incoming scattered wave $\Phi^{(\mathrm{sc})-}$ instead of outgoing $\Phi^{(\mathrm{sc})+}$ which is inconsistent with the boundary condition (5) we started from. Second, only by using $\Psi^{-}$, we are able to introduce the breakup amplitude in a standard form.

Thus, making use of the spectral decomposition for the three-body Green's function $G^{+}$in Eq. (12) in terms of the three-body scattering wave function $\Psi^{-}$, we arrive at

$$
\begin{aligned}
\Phi_{\boldsymbol{k}_{\alpha}, \boldsymbol{q}_{\alpha}}^{(\mathrm{sc})+}\left(\boldsymbol{r}_{\alpha}, \boldsymbol{\rho}_{\alpha}\right)= & \int d \boldsymbol{r}_{\alpha}^{\prime} d \boldsymbol{\rho}_{\alpha}^{\prime} \frac{d \boldsymbol{k}_{\alpha}^{\prime}}{(2 \pi)^{3}} \frac{d \boldsymbol{q}_{\alpha}^{\prime}}{(2 \pi)^{3}} \\
& \times \frac{\Psi_{\boldsymbol{k}_{\alpha}^{\prime}, \boldsymbol{q}_{\alpha}^{\prime}}^{-}\left(\boldsymbol{r}_{\alpha}, \boldsymbol{\rho}_{\alpha}\right) \Psi_{\boldsymbol{k}_{\alpha}^{\prime}, \boldsymbol{q}_{\alpha}^{\prime}}^{-*}\left(\boldsymbol{r}_{\alpha}^{\prime}, \boldsymbol{\rho}_{\alpha}^{\prime}\right)}{E-k_{\alpha}^{\prime 2} / 2 \mu_{\alpha}-q_{\alpha}^{\prime 2} / 2 M_{\alpha}+i 0} \\
& \times \bar{V}_{\alpha} \Phi_{n_{i}, \boldsymbol{q}_{i}}^{(i)}\left(\boldsymbol{r}_{\alpha}^{\prime}, \boldsymbol{\rho}_{\alpha}^{\prime}\right)+\cdots,
\end{aligned}
$$

where the dots indicate all possible two- and three-body bound states of the Hamiltonian $H$.

Let us define an amplitude

$$
f\left(\boldsymbol{k}_{\alpha}, \boldsymbol{q}_{\alpha}\right)=\int d \boldsymbol{r}_{\alpha} d \boldsymbol{\rho}_{\alpha} \Psi_{\boldsymbol{k}_{\alpha}, \boldsymbol{q}_{\alpha}}^{-*}\left(\boldsymbol{r}_{\alpha}, \boldsymbol{\rho}_{\alpha}\right) \bar{V}_{\alpha} \Phi_{n_{i}, \boldsymbol{q}_{i}}^{(i)}\left(\boldsymbol{r}_{\alpha}, \boldsymbol{\rho}_{\alpha}\right),
$$

and rewrite Eq. (13) in the form

$$
\begin{aligned}
\Phi_{\boldsymbol{k}_{\alpha}, \boldsymbol{q}_{\alpha}}^{(\mathrm{sc})+}\left(\boldsymbol{r}_{\alpha}, \boldsymbol{\rho}_{\alpha}\right)= & \int \frac{d \boldsymbol{k}_{\alpha}^{\prime}}{(2 \pi)^{3}} \frac{d \boldsymbol{q}_{\alpha}^{\prime}}{(2 \pi)^{3}} \\
& \times \frac{f\left(\boldsymbol{k}_{\alpha}^{\prime}, \boldsymbol{q}_{\alpha}^{\prime}\right) \Psi_{\boldsymbol{k}_{\alpha}^{\prime}, \boldsymbol{q}_{\alpha}^{\prime}}^{-}\left(\boldsymbol{r}_{\alpha}, \boldsymbol{\rho}_{\alpha}\right)}{E-k^{\prime 2}{ }_{\alpha} / 2 \mu_{\alpha}-q^{\prime 2}{ }_{\alpha} / 2 M_{\alpha}+i 0}+\cdots
\end{aligned}
$$

We shall show in the sequel that Eq. (14) is the desired breakup amplitude. Equation (15) establishes a relationship between the total wave function of any $2 \rightarrow 3$ breakup process in a Coulomb three-body system and wave function of the $3 \rightarrow 3$ process of scattering of all three particles of the system in the continuum through the corresponding breakup amplitude.

Let us call $\Omega_{0}$ the asymptotic domain, where all interparticle distances are large, i.e, $r_{\alpha} \rightarrow \infty, \rho_{\alpha} \rightarrow \infty$, so that $r_{\alpha} / \rho_{\alpha}$ is nonzero. In addition, we call $\Omega_{\alpha}$ the asymptotic regime, where $\rho_{\alpha} \rightarrow \infty$, however $r_{\alpha}$ satisfies the constraint $r_{\alpha} / \rho_{\alpha}$ $\rightarrow 0$. We also introduce the following notations for asymptotic forms of $\Phi^{(\mathrm{sc})+}$ and $\Psi^{-}$in $\Omega_{\nu}, \nu=0, \alpha$ :

$$
\begin{gathered}
\Phi_{\boldsymbol{k}_{\alpha}, \boldsymbol{q}_{\alpha}}^{(\mathrm{scc}+}\left(\boldsymbol{r}_{\alpha}, \boldsymbol{\rho}_{\alpha}\right) \stackrel{\Omega_{\nu}}{\rightarrow} \Phi_{\boldsymbol{k}_{\alpha}, \boldsymbol{q}_{\alpha}}^{(\nu)+}\left(\boldsymbol{r}_{\alpha}, \boldsymbol{\rho}_{\alpha}\right), \\
\Psi_{\boldsymbol{k}_{\alpha}, \boldsymbol{q}_{\alpha}}^{-}\left(\boldsymbol{r}_{\alpha}, \boldsymbol{\rho}_{\alpha}\right) \rightarrow \Psi_{\boldsymbol{k}_{\alpha}, \boldsymbol{q}_{\alpha}}^{(\nu)-}\left(\boldsymbol{r}_{\alpha}, \boldsymbol{\rho}_{\alpha}\right) .
\end{gathered}
$$

We find it convenient to denote by $\Psi^{(\alpha)-}$ only that part of the total wave function $\Psi^{-}$in the domain $\Omega_{\alpha}$ which corresponds to the case of all the particles in continuum. Possible two-fragment channel contributions will be singled out and dealt with separately. 
Since in the $\Omega_{0}$ domain all components of $\Psi^{-}$involving two-body and three-body bound states have an exponentially decreasing contribution, all the contribution to $\Phi^{\text {(sc) + }}$ comes from the continuum part of $\Psi^{-}$. Therefore, we get from Eq. (15), a fundamental asymptotic relationship

$$
\begin{aligned}
\Phi_{\boldsymbol{k}_{\alpha}, \boldsymbol{q}_{\alpha}}^{(0)+}\left(\boldsymbol{r}_{\alpha}, \boldsymbol{\rho}_{\alpha}\right)= & \int \frac{d \boldsymbol{k}_{\alpha}^{\prime}}{(2 \pi)^{3}} \frac{d \boldsymbol{q}_{\alpha}^{\prime}}{(2 \pi)^{3}} \\
& \times \frac{f\left(\boldsymbol{k}_{\alpha}^{\prime}, \boldsymbol{q}_{\alpha}^{\prime}\right) \Psi_{\boldsymbol{k}_{\alpha}^{\prime}, \boldsymbol{q}_{\alpha}^{\prime}}^{(0)-}\left(\boldsymbol{r}_{\alpha}, \boldsymbol{\rho}_{\alpha}\right)}{E-k^{\prime 2}{ }_{\alpha} / 2 \mu_{\alpha}-q^{\prime 2}{ }_{\alpha} / 2 M_{\alpha}+i 0} .
\end{aligned}
$$

Let us turn now to the case when particles $\beta$ and $\gamma$ remain close to each other. All components of $\Psi^{-}$involving threeparticle bound states decrease exponentially in the $\Omega_{\alpha}$ domain as well. However, here we have to retain the contribution from all two-cluster channels containing bound states of pair $(\beta, \gamma)$ which singles out from the total wave function $\Psi^{-}$in two-fragment asymptotic channel $\alpha$. Thus, one can write from Eq. (15) another asymptotic relationship

$$
\begin{aligned}
\Phi_{\boldsymbol{k}_{\alpha}, \boldsymbol{q}_{\alpha}}^{(\alpha)+}\left(\boldsymbol{r}_{\alpha}, \boldsymbol{\rho}_{\alpha}\right)= & \int \frac{d \boldsymbol{k}_{\alpha}^{\prime}}{(2 \pi)^{3}} \frac{d \boldsymbol{q}_{\alpha}^{\prime}}{(2 \pi)^{3}} \\
& \times \frac{f\left(\boldsymbol{k}_{\alpha}^{\prime}, \boldsymbol{q}_{\alpha}^{\prime}\right) \Psi_{\boldsymbol{k}_{\alpha}^{\prime}, \boldsymbol{q}_{\alpha}^{\prime}}^{(\alpha)-}\left(\boldsymbol{r}_{\alpha}, \boldsymbol{\rho}_{\alpha}\right)}{E-k^{\prime 2}{ }_{\alpha} / 2 \mu_{\alpha}-q^{\prime 2}{ }_{\alpha} / 2 M_{\alpha}+i 0} \\
& +\sum_{n_{\alpha}} \int \frac{d \boldsymbol{q}_{\alpha}^{\prime}}{(2 \pi)^{3}} \frac{f_{n_{\alpha}}\left(\boldsymbol{q}_{\alpha}^{\prime}\right) \Phi_{n_{\alpha}, q_{\alpha}^{\prime}}^{(\alpha)}\left(\boldsymbol{r}_{\alpha}, \boldsymbol{\rho}_{\alpha}\right)}{E-E_{n_{\alpha}}-q^{\prime 2}{ }_{\alpha} / 2 M_{\alpha}+i 0},
\end{aligned}
$$

where $f_{n_{\alpha}}$ is the amplitude for excitation of the two-fragment channel state $n_{\alpha}$. In the second term, the integral over $\boldsymbol{k}_{\alpha}^{\prime}$ became a sum over all energetically possible two-particle discrete states. The wave function of the two-fragment channel state $\Phi_{n_{\alpha}, q_{\alpha}}^{(\alpha)}$ is determined from Eqs. (6)-(8) with $n_{i}$ and $\boldsymbol{q}_{i}$ replaced by $n_{\alpha}$ and $\boldsymbol{q}_{\alpha}$, respectively.

Asymptotic relationships (18) and (19) were used to calculate forms of the scattered wave for electron-hydrogen scattering [23] valid in all asymptotic domains relevant to ionization. We note that the second term of Eq. (19) is familiar in the theory of potential scattering and leads to a known scattered wave. Different approximate relationships resembling Eq. (19) have been in use, e.g., in the close-coupling formalism for a long time. However, we emphasize that Eqs. (18) and (19) are exact.

\section{ASYMPTOTIC FORMS OF THE SCATTERED WAVE FOR BREAKUP}

In this section, we investigate the asymptotic behavior of the scattered wave $\Phi^{(\mathrm{sc})+}$ for a system of three arbitrary charged particles and calculate its leading-order terms in $\Omega_{0}$ and $\Omega_{\alpha}$ based on the relationships (18) and (19). Below we refer to asymptotic wave functions $\Phi^{(\nu)+}$ and $\Psi^{(\nu)+}, \nu$
$=0, \alpha$, to denote the leading-order terms of the relevant wave functions.

To this end, we need leading-order asymptotic terms of $\Psi^{-}$. For $\Omega_{0}$, in nonsingular directions $\left(\hat{\boldsymbol{k}}_{\nu} \cdot \hat{\boldsymbol{r}}_{\nu} \neq-1, \nu\right.$ $=\alpha, \beta, \gamma)$, this term was given by Redmond [30] and has the form of the three-body plane wave distorted by the longrange Coulomb interaction between all three pairs of particles:

$$
\Psi_{\boldsymbol{k}_{\alpha}, \boldsymbol{q}_{\alpha}}^{(0)-}\left(\boldsymbol{r}_{\alpha}, \boldsymbol{\rho}_{\alpha}\right)=e^{i \boldsymbol{k}_{\alpha} \cdot \boldsymbol{r}_{\alpha}+i \boldsymbol{q}_{\alpha} \cdot \boldsymbol{\rho}_{\alpha}} \prod_{\nu=\alpha, \beta, \gamma} e^{-i \eta_{\nu} / k_{\nu} \ln \zeta\left(\boldsymbol{k}_{\nu}, \boldsymbol{r}_{\nu}\right)},
$$

where

$$
\eta_{\alpha}=z_{\beta} z_{\gamma} \mu_{\alpha}, \quad \zeta\left(\boldsymbol{k}_{\alpha}, \boldsymbol{r}_{\alpha}\right)=k_{\alpha} r_{\alpha}+\boldsymbol{k}_{\alpha} \cdot \boldsymbol{r}_{\alpha} .
$$

For $\Omega_{\alpha}$, the leading asymptotic terms were obtained by Alt and Mukhamedzhanov [27] (AM):

$$
\begin{aligned}
\Psi_{\boldsymbol{k}_{\alpha}, \boldsymbol{q}_{\alpha}}^{(\alpha)-}\left(\boldsymbol{r}_{\alpha}, \boldsymbol{\rho}_{\alpha}\right)= & e^{i \boldsymbol{k}_{\alpha} \cdot \boldsymbol{r}_{\alpha}+i \boldsymbol{q}_{\alpha} \cdot \boldsymbol{\rho}_{\alpha}} \psi_{\alpha}\left(\widetilde{\boldsymbol{k}}_{\alpha}, \boldsymbol{r}_{\alpha}\right) \\
& \times \prod_{\nu=\beta, \gamma} e^{-i \eta_{\nu} / k_{\nu} \ln \zeta\left(\boldsymbol{k}_{\nu}, \boldsymbol{r}_{\nu}\right)} .
\end{aligned}
$$

The wave function $\psi_{\alpha}\left(\widetilde{\boldsymbol{k}}_{\alpha}, \boldsymbol{r}_{\alpha}\right)$ satisfies the following equation:

$$
\left[\frac{1}{2 \mu_{\alpha}} \Delta_{r_{\alpha}}+i \frac{1}{\mu_{\alpha}} \widetilde{\boldsymbol{k}}_{\alpha} \cdot \nabla_{r_{\alpha}}-V_{\alpha}\left(\boldsymbol{r}_{\alpha}\right)\right] \psi_{\alpha}\left(\widetilde{\boldsymbol{k}}_{\alpha}, \boldsymbol{r}_{\alpha}\right)=0
$$

with the incoming-wave boundary condition and describes the relative motion of particles $\beta$ and $\gamma$, interacting via the potential given by the sum of the Coulomb and short-range potentials $V_{\alpha}=V_{\alpha}^{C}+V_{\alpha}^{S}$. If the potential $V_{\alpha}$ is pure Coulombic, $V_{\alpha}=V_{\alpha}^{C}$, then $\psi_{\alpha}\left(\widetilde{\boldsymbol{k}}_{\alpha}, \boldsymbol{r}_{\alpha}\right)$ is given by

$$
\begin{aligned}
\psi_{\alpha}\left(\widetilde{\boldsymbol{k}}_{\alpha}, \boldsymbol{r}_{\alpha}\right)= & \Gamma\left(1-i \eta_{\alpha} / \widetilde{k}_{\alpha}\right) \exp \left(-\pi \eta_{\alpha} / 2 \widetilde{k}_{\alpha}\right)_{1} F_{1}\left(i \eta_{\alpha} / \widetilde{k}_{\alpha}, 1\right. \\
& \left.-i \zeta\left(\widetilde{\boldsymbol{k}}_{\alpha}, \boldsymbol{r}_{\alpha}\right)\right),
\end{aligned}
$$

where ${ }_{1} F_{1}$ is the confluent hypergeometric function. The relative local momentum $\widetilde{\boldsymbol{k}}_{\alpha}$ of particles $\beta$ and $\gamma$ in the Coulomb field of the third particle is given by

$$
\widetilde{\boldsymbol{k}}_{\alpha}=\boldsymbol{k}_{\alpha}+\sum_{\nu=\beta, \gamma} \frac{m_{\nu}}{m_{\beta}+m_{\gamma}} \frac{\eta_{\nu}}{k_{\nu}} \frac{\hat{\boldsymbol{k}}_{\nu}+\hat{\boldsymbol{r}}_{\nu}}{1+\hat{\boldsymbol{k}}_{\nu} \cdot \hat{\boldsymbol{r}}_{\nu}} \frac{1}{r_{\nu}} .
$$

Thus, the relative motion of particles $\beta$ and $\gamma$ is correlated by particle $\alpha$ at infinity due to the long-range nature of the Coulomb interaction. The importance of this three-body effect was first demonstrated practically [23] in the case of electron-impact ionization. The effect provided consistency of the underlying scattering theory, for instance, when two electrons are close to each other.

When $\Omega_{\alpha} \rightarrow \Omega_{0}$, the AM function smoothly transforms to the Redmond function as the local corrections in momentum $\tilde{\boldsymbol{k}}_{\alpha}$ become negligible. All second-order terms of $\Psi^{-}$in $\Omega_{\alpha}$ have been found by Mukhamedzhanov and Lieber [31]. 
However, as we are interested in this work, in the main leading-order terms of the scattered wave, the AM wave function is sufficient for our purpose.

Let us proceed now to the asymptotic behavior of the scattered wave $\Phi^{(\mathrm{sc})+}$. The standard procedure due to $\mathrm{Pe}$ terkop $[17,18]$ is to write Eq. (1) in terms of six-dimensional hyperspherical coordinates. Then, in $\Omega_{0}$, the Schrödinger equation (1) transforms into the Hamilton-Jacobi-type equation as the motion of the particles becomes classical. The Peterkop wave function was originally given for the case of two light particles in the Coulomb field of the infinitely heavy third particle. For further reference, here we give a similar wave function for the case of three arbitrary Coulomb particles. Thus, following Peterkop's procedure, we get, to leading order,

$$
\Phi_{\boldsymbol{k}_{\alpha}, \boldsymbol{q}_{\alpha}}^{(0)+}\left(\boldsymbol{r}_{\alpha}, \boldsymbol{\rho}_{\alpha}\right)=A(\hat{\omega}) R^{-5 / 2} e^{i \kappa R-i \lambda_{0} \ln (2 \kappa R)},
$$

where

$$
R=\left(\frac{\mu_{\alpha}}{m} r_{\alpha}^{2}+\frac{M_{\alpha}}{m} \rho_{\alpha}^{2}\right)^{1 / 2}
$$

is a hyperradius, $m$ is an arbitrary mass constant introduced for convenience so that the hyper-radius has units of length [35], $\hat{\omega}=\left(\hat{\boldsymbol{r}}_{\alpha}, \hat{\boldsymbol{\rho}}_{\alpha}, \varphi_{\alpha}\right)$ is a five-dimensional hyperangle, with

$$
\varphi_{\alpha}=\arctan \left[\left(\frac{\mu_{\alpha}}{M_{\alpha}}\right)^{1 / 2} \frac{r_{\alpha}}{\rho_{\alpha}}\right], \quad 0 \leqslant \varphi_{\alpha} \leqslant \pi / 2,
$$

$\kappa=(2 m E)^{1 / 2}$, and the Coulomb parameter $\lambda_{0}$ is given by

$$
\lambda_{0}=\frac{1}{\kappa} \sum_{\nu=\alpha, \beta, \gamma}\left(\frac{m}{\mu_{\nu}}\right)^{1 / 2} \frac{\eta_{\nu}}{\sin \varphi_{\nu}} .
$$

$A(\hat{\omega})$ is the breakup amplitude. For brevity of representation, in Eq. (29), we introduced additional hyperangles $\varphi_{\nu}, \nu$ $=\beta, \gamma$. These are defined similar to $\varphi_{\alpha}[$ see Eq. (28)] and related to $\varphi_{\alpha}$ according to

$$
\begin{aligned}
\sin \varphi_{\nu}= & {\left[\frac{\mu_{\nu}}{M_{\alpha}} \cos ^{2} \varphi_{\alpha}+\frac{\mu_{\nu} \mu_{\alpha}}{m_{\gamma}^{2}} \sin ^{2} \varphi_{\alpha}\right.} \\
& \left.+\epsilon_{\nu \alpha} \frac{\mu_{\nu}}{m_{\gamma}}\left(\frac{\mu_{\alpha}}{M_{\alpha}}\right)^{1 / 2} \sin 2 \varphi_{\alpha} \hat{\boldsymbol{r}}_{\alpha} \cdot \hat{\boldsymbol{\rho}}_{\alpha}\right]^{1 / 2}, \quad \nu=\beta, \gamma .
\end{aligned}
$$

Equation (29) explicitly shows that the generalized Peterkop wave function given by Eq. (26) is not valid in the regions where $\varphi_{\nu} \rightarrow 0, \nu=\alpha, \beta, \gamma$, i.e., when any two particles of the system are close to each other and far from the third one. The main drawback of the Peterkop asymptotic form, however, lies in an amplitude-phase ambiguity problem, when some part of $A(\hat{\omega})$ can be moved to the phase factor and the resulting wave function is still a solution to the original Hamilton-Jacobi equation [15]. Accordingly, remainder $A^{\prime}(\hat{\omega})$ can equally well be called a breakup amplitude. Thus, generally speaking, the hyperspherical approach is not capable of uniquely identifying the breakup amplitude. Our approach will enable us to fix this problem, unambiguously relating the "hyperspherical" definition of the breakup amplitude to its standard quantum-mechanical one given by Eq. (14).

Let us now calculate the same wave function $\Phi^{(0)+}$ using the relationship (18) and noting the leading-order asymptotic terms given in Eqs. (20)-(25). Using the asymptotic forms makes it possible to evaluate Eq. (18). To this end, we consider first the integral over $\boldsymbol{k}_{\alpha}^{\prime}$ :

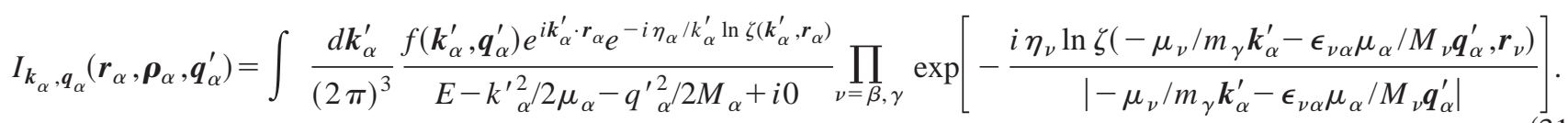

We take advantage of the fact that in $\Omega_{0}$ domain $r_{\alpha} \rightarrow \infty$ and use an asymptotic form of the plane wave

$$
e^{i \boldsymbol{k} \cdot \boldsymbol{r}} \sim \stackrel{2 \pi}{i k r}\left[\delta(\hat{\boldsymbol{k}}-\hat{\boldsymbol{r}}) e^{i k r}-\delta(\hat{\boldsymbol{k}}+\hat{\boldsymbol{r}}) e^{-i k r}\right]
$$

which can be obtained from the asymptotic form of the partial wave expansion of the plane wave (see, e.g., Ref. [32]). Then we get (in the leading order)

$$
\begin{aligned}
& I_{\boldsymbol{k}_{\alpha}, \boldsymbol{q}_{\alpha}}\left(\boldsymbol{r}_{\alpha}, \boldsymbol{\rho}_{\alpha}, \boldsymbol{q}_{\alpha}^{\prime}\right)=\frac{1}{(2 \pi)^{2}} \frac{1}{i r_{\alpha}} \int_{-\infty}^{\infty} d k_{\alpha}^{\prime} \frac{k_{\alpha}^{\prime} f\left(k_{\alpha}^{\prime} \hat{\boldsymbol{r}}_{\alpha}, \boldsymbol{q}_{\alpha}^{\prime}\right) e^{i k_{\alpha}^{\prime} r_{\alpha}} e^{-i \eta_{\alpha} / k_{\alpha}^{\prime} \ln \zeta\left(k_{\alpha}^{\prime} \hat{\boldsymbol{r}}_{\alpha}, \boldsymbol{r}_{\alpha}\right)}}{E-k_{\alpha}^{\prime 2} / 2 \mu_{\alpha}-q_{\alpha}^{\prime 2} / 2 M_{\alpha}+i 0} \\
& \times \prod_{\nu=\beta, \gamma} \exp \left[-\frac{i \eta_{\nu} \ln \zeta\left(-\mu_{\nu} / m_{\gamma} k_{\alpha}^{\prime} \hat{\boldsymbol{r}}_{\alpha}-\boldsymbol{\epsilon}_{\nu \alpha} \mu_{\alpha} / M_{\nu} \boldsymbol{q}_{\alpha}^{\prime}, \boldsymbol{r}_{\nu}\right)}{\left|-\mu_{\nu} / m_{\gamma} k_{\alpha}^{\prime} \hat{\boldsymbol{r}}_{\alpha}-\boldsymbol{\epsilon}_{\nu \alpha} \mu_{\alpha} / M_{\nu} \boldsymbol{q}_{\alpha}^{\prime}\right|}\right] .
\end{aligned}
$$


The integrand has two simple poles and apart from that it is an analytic function on the complex energy plane. Therefore, we can calculate this integral by closing the integration contour, e.g., in the upper half of the complex plane (semicircle complex contour of infinite radius does not contribute to the integral due to the $e^{i k_{\alpha}^{\prime} r_{\alpha}}$ factor), and using the Cauchy theorem to take residue at the pole singularity $(\mathrm{ps})$ :

$$
\begin{aligned}
I_{\boldsymbol{k}_{\alpha}, \boldsymbol{q}_{\alpha}}\left(\boldsymbol{r}_{\alpha}, \boldsymbol{\rho}_{\alpha}, \boldsymbol{q}_{\alpha}^{\prime}\right)= & -\frac{\mu_{\alpha}}{2 \pi} \frac{e^{i k_{\alpha}^{(\mathrm{ps})} r_{\alpha}}}{r_{\alpha}} f\left(k_{\alpha}^{(\mathrm{ps})} \hat{\boldsymbol{r}}_{\alpha}, \boldsymbol{q}_{\alpha}^{\prime}\right) e^{-i \eta_{\alpha} / k_{\alpha}^{(\mathrm{ps})} \ln \zeta\left(k_{\alpha}^{(\mathrm{ps})} \hat{\boldsymbol{r}}_{\alpha}, \boldsymbol{r}_{\alpha}\right)} \\
& \times \prod_{\nu=\beta, \gamma} \exp \left[-\frac{i \eta_{\nu} \ln \zeta\left(-\mu_{\nu} / m_{\gamma} k_{\alpha}^{(\mathrm{ps})} \hat{\boldsymbol{r}}_{\alpha}-\boldsymbol{\epsilon}_{\nu \alpha} \mu_{\alpha} / M_{\nu} \boldsymbol{q}_{\alpha}^{\prime}, \boldsymbol{r}_{\nu}\right)}{\left|-\mu_{\nu} / m_{\gamma} k_{\alpha}^{(\mathrm{ps})} \hat{\boldsymbol{r}}_{\alpha}-\boldsymbol{\epsilon}_{\nu \alpha} \mu_{\alpha} / M_{\nu} \boldsymbol{q}_{\alpha}^{\prime}\right|}\right]
\end{aligned}
$$

This brings the energy conservation into play and the magnitude of $k_{\alpha}^{\prime}$ is now fixed at

$$
k_{\alpha}^{(\mathrm{ps})}=\left(2 \mu_{\alpha} E-\frac{\mu_{\alpha}}{M_{\alpha}} q_{\alpha}^{\prime 2}\right)^{1 / 2}
$$

Thus, we have

$$
\begin{aligned}
& \Phi_{\boldsymbol{k}_{\alpha}, \boldsymbol{q}_{\alpha}}^{(0)+}\left(\boldsymbol{r}_{\alpha}, \boldsymbol{\rho}_{\alpha}\right)=\int \frac{d \boldsymbol{q}_{\alpha}^{\prime}}{(2 \pi)^{3}} e^{i \boldsymbol{q}_{\alpha}^{\prime} \cdot \boldsymbol{\rho}_{\alpha}} I_{\boldsymbol{k}_{\alpha}, \boldsymbol{q}_{\alpha}}\left(\boldsymbol{r}_{\alpha}, \boldsymbol{\rho}_{\alpha}, \boldsymbol{q}_{\alpha}^{\prime}\right)
\end{aligned}
$$

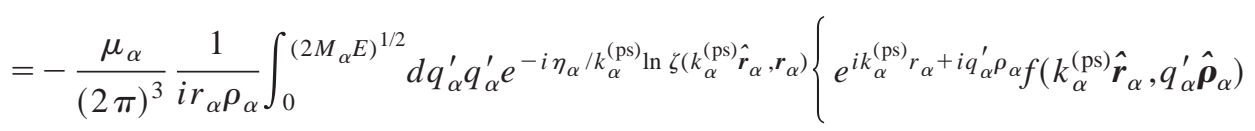

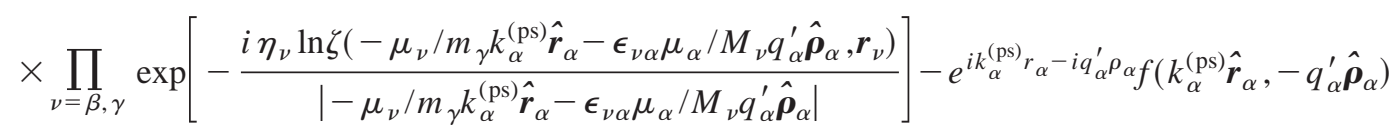

$$
\begin{aligned}
& \left.\times \prod_{\nu=\beta, \gamma} \exp \left[-\frac{i \eta_{\nu} \ln \zeta\left(-\mu_{\nu} / m_{\gamma} k_{\alpha}^{(\mathrm{ps})} \hat{\boldsymbol{r}}_{\alpha}+\boldsymbol{\epsilon}_{\nu \alpha} \mu_{\alpha} / M_{\nu} q_{\alpha}^{\prime} \hat{\boldsymbol{\rho}}_{\alpha}, \boldsymbol{r}_{\nu}\right)}{\left|-\mu_{\nu} / m_{\gamma} k_{\alpha}^{(\mathrm{ps})} \hat{\boldsymbol{r}}_{\alpha}+\boldsymbol{\epsilon}_{\nu \alpha} \mu_{\alpha} / M_{\nu} q_{\alpha}^{\prime} \hat{\boldsymbol{\rho}}_{\alpha}\right|}\right]\right\} .
\end{aligned}
$$

At this stage, we have no information about the individual physical momenta $\boldsymbol{k}_{\alpha}$ and $\boldsymbol{q}_{\alpha}$ but, as we will presently show, their values will become apparent upon evaluating the integral using asymptotic techniques. In $\Omega_{0}$, where $r_{\alpha}$ and $\rho_{\alpha}$ are asymptotically large, the integrand is extremely oscillatory. For this reason, one should expect any significant contribution to the integral only from neighborhood of stationary-phase ( $\mathrm{sp}$ ) points if there are any. One can verify that the first term of the integrand in Eq. (36) has a single stationary-phase point at

$$
q_{\alpha}^{(\mathrm{sp})}=\frac{M_{\alpha}}{m} \frac{\kappa}{R} \rho_{\alpha}
$$

while the second one does not have any. This is why, a contribution to the integral from the second term in curly brackets is negligibly small. Evaluating the remaining integral by means of the stationary-phase method [33], we obtain

$$
\begin{aligned}
\Phi_{\boldsymbol{k}_{\alpha}, \boldsymbol{q}_{\alpha}}^{(0)+}\left(\boldsymbol{r}_{\alpha}, \boldsymbol{\rho}_{\alpha}\right)= & \frac{(2 \pi i)^{1 / 2}}{(2 \pi)^{3}} f\left(\frac{\mu_{\alpha}}{m} \frac{\kappa}{R} \boldsymbol{r}_{\alpha}, \frac{M_{\alpha}}{m} \frac{\kappa}{R} \boldsymbol{\rho}_{\alpha}\right) \\
& \times \frac{\left(\mu_{\alpha} M_{\alpha}\right)^{3 / 2}}{m^{2}} \frac{\kappa^{3 / 2}}{R^{5 / 2}} e^{i \kappa R} \\
& \times \prod_{\nu=\alpha, \beta, \gamma} \exp \left[-\frac{i \eta_{\nu} m R}{\mu_{\nu} \kappa r_{\nu}} \ln \left(\frac{2 \mu_{\nu}}{m} \frac{\kappa}{R} r_{\nu}^{2}\right)\right] .
\end{aligned}
$$

In Eq. (38), we used the fact that at the stationary-phase point, Eq. (35) is written as

$$
k_{\alpha}^{(\mathrm{sp})}=\frac{\mu_{\alpha}}{m} \frac{\kappa}{R} r_{\alpha},
$$

and consequently 


$$
-\frac{\mu_{\nu}}{m_{\gamma}} k_{\alpha}^{(\mathrm{ps})} \hat{\boldsymbol{r}}_{\alpha}-\epsilon_{\nu \alpha} \frac{\mu_{\alpha}}{M_{\nu}} q_{\alpha}^{(\mathrm{sp})} \hat{\boldsymbol{\rho}}_{\alpha}=\frac{\mu_{\nu}}{m} \frac{\kappa}{R} \boldsymbol{r}_{\nu}, \quad \nu=\beta, \gamma
$$

We also note that the physical momenta $\boldsymbol{k}_{\alpha}$ and $\boldsymbol{q}_{\alpha}$ are given by

$$
\boldsymbol{k}_{\alpha}=\frac{\mu_{\alpha}}{m} \frac{\kappa}{R} \boldsymbol{r}_{\alpha}, \quad \boldsymbol{q}_{\alpha}=\frac{M_{\alpha}}{m} \frac{\kappa}{R} \boldsymbol{\rho}_{\alpha}
$$

In terms of hyperangles $\varphi_{\nu}, \nu=\alpha, \beta, \gamma$, we finally have

$$
\begin{aligned}
\Phi_{k_{\alpha}, q_{\alpha}}^{(0)+}\left(\boldsymbol{r}_{\alpha}, \boldsymbol{\rho}_{\alpha}\right)= & \frac{(2 \pi i)^{1 / 2}}{(2 \pi)^{3}} f\left(\frac{\mu_{\alpha}}{m} \frac{\kappa}{R} \boldsymbol{r}_{\alpha} \frac{M_{\alpha}}{m} \frac{\kappa}{R} \boldsymbol{\rho}_{\alpha}\right) \\
& \times \frac{\left(\mu_{\alpha} M_{\alpha}\right)^{3 / 2}}{m^{2}} \frac{\kappa^{3 / 2}}{R^{5 / 2}} \\
& \times \exp \left[i \kappa R-i \lambda_{0} \ln (2 \kappa R)-i \sigma_{0}\right],
\end{aligned}
$$

with the additional phase

$$
\sigma_{0}=\frac{2}{\kappa} \sum_{\nu=\alpha, \beta, \gamma}\left(\frac{m}{\mu_{\nu}}\right)^{1 / 2} \frac{\eta_{\nu} \ln \left(\sin \varphi_{\nu}\right)}{\sin \varphi_{\nu}}
$$

Thus, the asymptotic form of $\Phi^{(\mathrm{sc})+}$ in $\Omega_{0}$ comes as a result of the fundamental relationship between the total wave functions describing two different scattering processes within the same three-body system. Most importantly, our derivation leads to an unambiguous amplitude-phase form, which allows us to uniquely express the hyperspherical breakup amplitude $A(\hat{\omega})$ in terms of the standard definition of the breakup amplitude $f\left(\boldsymbol{k}_{\alpha}, \boldsymbol{q}_{\alpha}\right)$ given by Eq. (14):

$$
\begin{aligned}
A(\hat{\omega})= & \frac{(2 \pi i)^{1 / 2}}{(2 \pi)^{3}} \frac{\left(\mu_{\alpha} M_{\alpha}\right)^{3 / 2}}{m^{2}} \\
& \times \kappa^{3 / 2} f\left(\frac{\mu_{\alpha}}{m} \frac{\kappa}{R} \boldsymbol{r}_{\alpha}, \frac{M_{\alpha}}{m} \frac{\kappa}{R} \boldsymbol{\rho}_{\alpha}\right) e^{-i \sigma_{0}} .
\end{aligned}
$$

Having completed the derivation of the asymptotic form of $\Phi_{\boldsymbol{k}_{\alpha}, q_{\alpha}}^{(0)+}\left(\boldsymbol{r}_{\alpha}, \boldsymbol{\rho}_{\alpha}\right)$ in $\Omega_{0}$, it remains to proceed to $\Omega_{\alpha}$ and evaluate the integrals contained in Eq. (19). To start with, we consider the second term of Eq. (19). The leading-order terms of the wave functions in the two-fragment channels, as $\rho_{\alpha} \rightarrow \infty$, are given by

$$
\Phi_{n_{\alpha}, \boldsymbol{q}_{\alpha}}^{(\alpha)}\left(\boldsymbol{r}_{\alpha}, \boldsymbol{\rho}_{\alpha}\right) \rightarrow e^{i \boldsymbol{q}_{\alpha} \cdot \boldsymbol{\rho}_{\alpha}-i \bar{\eta}_{\alpha} / q_{\alpha} \ln \zeta\left(\boldsymbol{q}_{\alpha}, \boldsymbol{\rho}_{\alpha}\right)} \phi_{n_{\alpha}}\left(\boldsymbol{r}_{\alpha}\right),
$$

with

$$
\bar{\eta}_{\alpha}=z_{\alpha}\left(z_{\beta}+z_{\gamma}\right) M_{\alpha} .
$$

Consequently, for the second term of Eq. (19), we have

$$
\begin{aligned}
\Phi_{\boldsymbol{k}_{\alpha}, \boldsymbol{q}_{\alpha}}^{(\alpha)+}\left(\boldsymbol{r}_{\alpha}, \boldsymbol{\rho}_{\alpha}\right) \sim & \sum_{n_{\alpha}} \int \frac{d \boldsymbol{q}_{\alpha}^{\prime}}{(2 \pi)^{3}} \\
& \times \frac{f_{n_{\alpha}}\left(\boldsymbol{q}_{\alpha}^{\prime}\right) e^{i \boldsymbol{q}_{\alpha}^{\prime} \cdot \boldsymbol{\rho}_{\alpha}-i \bar{\eta}_{\alpha} / q_{\alpha}^{\prime} \ln \zeta\left(\boldsymbol{q}_{\alpha}^{\prime}, \boldsymbol{\rho}_{\alpha}\right)} \phi_{n_{\alpha}}\left(\boldsymbol{r}_{\alpha}\right)}{E-E_{n_{\alpha}}-q^{\prime 2}{ }_{\alpha} / 2 M_{\alpha}+i 0} .
\end{aligned}
$$

The calculation of the integral in Eq. (47) for large $\rho_{\alpha}$ is trivial and leads to well-known outgoing scattered waves in two-cluster channels. However, we keep this term for completeness of the final result.

Consider now the first term of Eq. (19). By definition, here $r_{\alpha}$ is limited as compared to $\rho_{\alpha}$. Therefore, it cannot, strictly speaking, be used as an asymptotic parameter alongside $\rho_{\alpha}$. However, the other two pairs of Jacobi variables $\left(\boldsymbol{r}_{\nu}, \boldsymbol{\rho}_{\nu}\right), \nu=\beta, \gamma$, constitute suitable pairs of asymptotically large parameters, should we represent the integral in terms of relevant canonical conjugate momentum space variables $\left(\boldsymbol{k}_{\nu}, \boldsymbol{q}_{\nu}\right)$. Below we use $\left(\boldsymbol{k}_{\beta}, \boldsymbol{q}_{\beta}\right)$ space. Then, for the first term of Eq. (19), we have

$$
\begin{aligned}
\Phi_{\boldsymbol{k}_{\alpha}, \boldsymbol{q}_{\alpha}}^{(\alpha)+}\left(\boldsymbol{r}_{\alpha}, \boldsymbol{\rho}_{\alpha}\right) \sim & \int \frac{d \boldsymbol{k}_{\beta}^{\prime}}{(2 \pi)^{3}} \frac{d \boldsymbol{q}_{\beta}^{\prime}}{(2 \pi)^{3}} \\
& \times \frac{f\left(\boldsymbol{k}_{\alpha}^{\prime}, \boldsymbol{q}_{\alpha}^{\prime}\right) e^{i \boldsymbol{k}_{\beta}^{\prime} \cdot \boldsymbol{r}_{\beta}+i \boldsymbol{q}_{\beta}^{\prime} \cdot \boldsymbol{\rho}_{\beta} \psi_{\alpha}\left(\widetilde{\boldsymbol{k}}_{\alpha}, \boldsymbol{r}_{\alpha}\right)}}{E-k^{\prime 2}{ }_{\beta} / 2 \mu_{\beta}-q^{\prime 2}{ }_{\beta} / 2 M_{\beta}+i 0} \\
& \times \prod_{\nu=\beta, \gamma} e^{-i \eta_{\nu} / k_{\nu} \ln \zeta\left(\boldsymbol{k}_{\nu}, \boldsymbol{r}_{\nu}\right)} .
\end{aligned}
$$

In the above equation $\boldsymbol{r}_{\nu}, \boldsymbol{\rho}_{\nu}, \boldsymbol{k}_{\nu}^{\prime}$, and $\boldsymbol{q}_{\nu}^{\prime}, \nu=\gamma, \alpha$, are kept as short-hand notations. As functions of $\beta$-space variables, they are given by Eqs. (1) and (2). Taking into account that

$$
\begin{aligned}
& \frac{\mu_{\beta}}{m} r_{\beta}^{2}+\frac{M_{\beta}}{m} \rho_{\beta}^{2}=\frac{\mu_{\alpha}}{m} r_{\alpha}^{2}+\frac{M_{\alpha}}{m} \rho_{\alpha}^{2}, \\
& \frac{m}{\mu_{\beta}} k_{\beta}^{2}+\frac{m}{M_{\beta}} q_{\beta}^{2}=\frac{m}{\mu_{\alpha}} k_{\alpha}^{2}+\frac{m}{M_{\alpha}} q_{\alpha}^{2},
\end{aligned}
$$

we can calculate the above integral in analogy with the procedure we used in $\Omega_{0}$. We therefore omit the details. Evaluating the integrals and transforming the answer back to the conventional for $\Omega_{\alpha}$ variables $\boldsymbol{r}_{\alpha}$ and $\boldsymbol{\rho}_{\alpha}$, and also adding the outcome from Eq. (47), we arrive at 


$$
\begin{aligned}
\Phi_{\boldsymbol{k}_{\alpha}, \boldsymbol{q}_{\alpha}}^{(\alpha)+}\left(\boldsymbol{r}_{\alpha}, \boldsymbol{\rho}_{\alpha}\right)= & \frac{(2 \pi i)^{1 / 2}}{(2 \pi)^{3}} f\left(\frac{\mu_{\alpha}}{m} \frac{\kappa}{R} \boldsymbol{r}_{\alpha}, \frac{M_{\alpha}}{m} \frac{\kappa}{R} \boldsymbol{\rho}_{\alpha}\right) \\
& \times \frac{\left(\mu_{\alpha} M_{\alpha}\right)^{3 / 2}}{m^{2}} \frac{\kappa^{3 / 2}}{R^{5 / 2}} \psi_{\alpha}\left(\boldsymbol{K}_{\alpha}, \boldsymbol{r}_{\alpha}\right) \\
& \times e^{i \kappa R-i \lambda_{\alpha} \ln (2 \kappa R)-i \sigma_{\alpha}} \\
& -\frac{M_{\alpha}}{2 \pi} \sum_{n_{\alpha}} f_{n_{\alpha}}\left(q_{n_{\alpha}} \hat{\boldsymbol{\rho}}_{\alpha}\right) \frac{e^{i q_{n_{\alpha}} \rho_{\alpha}}}{\rho_{\alpha}} \\
& \times e^{-i \bar{\eta}_{\alpha} / q_{n_{\alpha}} \ln \left(2 q_{n_{\alpha}} \rho_{\alpha}\right)} \boldsymbol{\phi}_{n_{\alpha}}\left(\boldsymbol{r}_{\alpha}\right),
\end{aligned}
$$

where

$$
\lambda_{\alpha}=\frac{1}{\kappa} \sum_{\nu=\beta, \gamma}\left(\frac{m}{\mu_{\nu}}\right)^{1 / 2} \frac{\eta_{\nu}}{\sin \varphi_{\nu}}
$$

and

$$
\sigma_{\alpha}=\frac{2}{\kappa} \sum_{\nu=\beta, \gamma}\left(\frac{m}{\mu_{\nu}}\right)^{1 / 2} \frac{\eta_{\nu} \ln \left(\sin \varphi_{\nu}\right)}{\sin \varphi_{\nu}}
$$

The new local momentum entering in the scattered wave (50) is given by

$$
\boldsymbol{K}_{\alpha}=\frac{\mu_{\alpha}}{m} \frac{\kappa}{R} \boldsymbol{r}_{\alpha}+\sum_{\nu=\beta, \gamma} \frac{m_{\nu}}{m_{\beta}+m_{\gamma}} \frac{\eta_{\nu}}{\mu_{\nu}} \frac{R}{\kappa r_{\nu}^{3}} \boldsymbol{r}_{\nu}
$$

The momentum of the scattered particle $\alpha$ relative to the bound pair $(\beta, \gamma)$ is given by

$$
q_{n_{\alpha}}=\left[2 M_{\alpha}\left(E-E_{n_{\alpha}}\right)\right]^{1 / 2} .
$$

The second term with $\phi_{n_{\alpha}}\left(\boldsymbol{r}_{\alpha}\right)$ in Eq. (50) disappears as $r_{\alpha} \rightarrow \infty$. In addition, if we take into account that as $r_{\alpha}$ grows large the second term in Eq. (53) becomes negligible, so that

$$
\psi_{\alpha}\left(\boldsymbol{K}_{\alpha}, \boldsymbol{r}_{\alpha}\right)^{r_{\alpha} \rightarrow \infty} \sim \exp \left[-\frac{i}{\kappa}\left(\frac{m}{\mu_{\alpha}}\right)^{1 / 2} \frac{\eta_{\alpha}}{\sin \varphi_{\alpha}} \ln \left(2 \kappa R \sin ^{2} \varphi_{\alpha}\right)\right] .
$$

All this means that Eq. (50) smoothly transforms to Eq. (42) when $\Omega_{\alpha} \rightarrow \Omega_{0}$.

It is now also not difficult to verify that the final results for the scattered wave are independent of the complementary mass constant $m$ which we introduced earlier.

Thus, the generalized asymptotic scattered wave valid in all the asymptotic regions relevant to breakup can be written as

$$
\begin{aligned}
\Phi_{\boldsymbol{k}_{\alpha}, \boldsymbol{q}_{\alpha}}^{(\mathrm{sc})+}\left(\boldsymbol{r}_{\alpha}, \boldsymbol{\rho}_{\alpha}\right) \rightarrow & \frac{1}{(2 \pi)^{5 / 2}} f\left(\frac{\mu_{\alpha}}{m} \frac{\kappa}{R} \boldsymbol{r}_{\alpha}, \frac{M_{\alpha}}{m} \frac{\kappa}{R} \boldsymbol{\rho}_{\alpha}\right) \\
& \times \frac{\left(\mu_{\alpha} M_{\alpha}\right)^{3 / 2}}{m^{2}} \frac{\kappa^{3 / 2}}{R^{5 / 2}} e^{i \kappa R+i \pi / 4} \\
& \times \prod_{\nu=\alpha, \beta, \gamma} \psi_{\nu}\left(\boldsymbol{K}_{\nu}, \boldsymbol{r}_{\nu}\right) \\
& -\frac{1}{2 \pi_{\nu}} \sum_{\alpha, \beta, \gamma} M_{\nu} \sum_{n_{\nu}} f_{n_{\nu}}\left(q_{n_{\nu}} \hat{\boldsymbol{\rho}}_{\nu}\right) \\
& \times \frac{e^{i q_{n_{\nu}} \rho_{\nu}}}{\rho_{\nu}} e^{-i \eta_{\nu} / q_{n_{\nu}} \ln \left(2 q_{n_{\nu}} \rho_{\nu}\right)} \phi_{n_{\nu}}\left(\boldsymbol{r}_{\nu}\right) .
\end{aligned}
$$

In $\Omega_{\alpha}$ Eq. (50') reduces to Eq. (50), while in $\Omega_{0}$ it is equivalent to Eq. (42).

\section{ASYMPTOTIC FORMS OF THE COULOMB THREE- BODY GREEN'S FUNCTION}

In this section, we state our results for the asymptotic forms of the Green's function for a system of three charged particles. Because the methods used earlier form the basis of the derivations, we will omit technical details of the calculations. Asymptotic forms of the three-body Green's function are important in the formulation of the three-body problem $[24,25]$ when calculating the optical potentials [26-28] and doing the nonperturbational calculations of dynamical dipole polarization terms [29].

Thus, using a similar technique, we can get leading-order terms of the three-body Green's function in asymptotic domains $\Omega_{0}$ and $\Omega_{\alpha}$. When $\left(r_{\alpha}, \rho_{\alpha}\right) \in \Omega_{0}$, from the spectral decomposition, we can write

$$
G^{+}\left(\boldsymbol{r}_{\alpha}, \boldsymbol{\rho}_{\alpha} ; \boldsymbol{r}_{\alpha}^{\prime}, \boldsymbol{\rho}_{\alpha}^{\prime} ; E+i 0\right) \stackrel{\left(r_{\alpha}, \rho_{\alpha}\right) \in \Omega_{0}}{\longrightarrow} \int \frac{d \boldsymbol{k}_{\alpha}^{\prime}}{(2 \pi)^{3}} \frac{d \boldsymbol{q}_{\alpha}^{\prime}}{(2 \pi)^{3}} \frac{\Psi_{\boldsymbol{k}_{\alpha}^{\prime}, q_{\alpha}^{\prime}}^{(0)-}\left(\boldsymbol{r}_{\alpha}, \boldsymbol{\rho}_{\alpha}\right) \Psi_{\boldsymbol{k}_{\alpha}^{\prime}, q_{\alpha}^{\prime}}^{-*}\left(\boldsymbol{r}_{\alpha}^{\prime}, \boldsymbol{\rho}_{\alpha}^{\prime}\right)}{E-k_{\alpha}^{\prime 2} / 2 \mu_{\alpha}-q_{\alpha}^{\prime 2}{ }_{\alpha} / 2 M_{\alpha}+i 0}
$$

Calculating the integrals, we get

$$
\begin{aligned}
G^{+}\left(\boldsymbol{r}_{\alpha}, \boldsymbol{\rho}_{\alpha} ; \boldsymbol{r}_{\alpha}^{\prime}, \boldsymbol{\rho}_{\alpha}^{\prime} ; E+i 0\right) \stackrel{\left(r_{\alpha}, \rho_{\alpha}\right) \in \Omega_{0}}{\longrightarrow} & \frac{(2 \pi i)^{1 / 2}}{(2 \pi)^{3}} \frac{\left(\mu_{\alpha} M_{\alpha}\right)^{3 / 2}}{m^{2}} \frac{\kappa^{3 / 2}}{R^{5 / 2}} \Psi_{\left(\mu_{\alpha} / m\right)(\kappa / R) \boldsymbol{r}_{\alpha},\left(M_{\alpha} / m\right)(\kappa / R) \boldsymbol{\rho}_{\alpha}}^{-*}\left(\boldsymbol{r}_{\alpha}^{\prime}, \boldsymbol{\rho}_{\alpha}^{\prime}\right) \\
& \times \exp \left[i \kappa R-i \lambda_{0} \ln (2 \kappa R)-i \sigma_{0}\right] .
\end{aligned}
$$

More interesting is the case when both $\left(r_{\alpha}, \rho_{\alpha}\right)$ and $\left(r_{\alpha}^{\prime}, \rho_{\alpha}^{\prime}\right) \in \Omega_{0}$. Then we have 


$$
\begin{aligned}
G^{+}\left(\boldsymbol{r}_{\alpha}, \boldsymbol{\rho}_{\alpha} ; \boldsymbol{r}_{\alpha}^{\prime}, \boldsymbol{\rho}_{\alpha}^{\prime} ; E+i 0\right) \stackrel{\begin{array}{c}
\left(r_{\alpha}, \rho_{\alpha}\right) \in \Omega_{0} \\
\left(r_{\alpha}^{\prime}, \rho_{\alpha}^{\prime}\right) \in \Omega_{0}
\end{array}}{\longrightarrow} & \frac{(2 \pi i)^{1 / 2}}{(2 \pi)^{3}} \frac{\left(\mu_{\alpha} M_{\alpha}\right)^{3 / 2}}{m^{2}} \frac{\kappa^{3 / 2}}{R^{5 / 2}} \exp \left[i \kappa R-i \kappa R^{\prime}\left(\sin \varphi_{\alpha} \sin \varphi_{\alpha}^{\prime} \hat{\boldsymbol{r}}_{\alpha} \cdot \hat{\boldsymbol{r}}_{\alpha}^{\prime}+\cos \varphi_{\alpha} \cos \varphi_{\alpha}^{\prime} \hat{\boldsymbol{\rho}}_{\alpha} \cdot \hat{\boldsymbol{\rho}}_{\alpha}^{\prime}\right)\right] \\
& \times \exp \left[-\frac{i}{\kappa} \sum_{\nu=\alpha, \beta, \gamma}\left(\frac{m}{\mu_{\nu}}\right)^{1 / 2} \frac{\eta_{\nu}}{\sin \varphi_{\nu}} \ln \frac{2 r_{\nu}}{r_{\nu}^{\prime}\left(1+\hat{\boldsymbol{r}}_{\nu} \cdot \hat{\boldsymbol{r}}_{\nu}^{\prime}\right)}\right]
\end{aligned}
$$

with the condition that $R^{\prime} \leqslant R$, otherwise the boundary condition for $G^{+}$is violated. The $R^{\prime}>R$ case defines $G^{-}$.

When $\left(r_{\alpha}, \rho_{\alpha}\right) \in \Omega_{\alpha}$, we can write

$$
\begin{aligned}
G^{+}\left(\boldsymbol{r}_{\alpha}, \boldsymbol{\rho}_{\alpha} ; \boldsymbol{r}_{\alpha}^{\prime}, \boldsymbol{\rho}_{\alpha}^{\prime} ; E+i 0\right) \stackrel{\left(r_{\alpha}, \rho_{\alpha}\right) \in \Omega_{\alpha}}{\longrightarrow} \int \frac{d \boldsymbol{k}_{\alpha}^{\prime}}{(2 \pi)^{3}} \frac{d \boldsymbol{q}_{\alpha}^{\prime}}{(2 \pi)^{3}} \frac{\Psi_{\boldsymbol{k}_{\alpha}^{\prime}, \boldsymbol{q}_{\alpha}^{\prime}}^{(\alpha)}\left(\boldsymbol{r}_{\alpha}, \boldsymbol{\rho}_{\alpha}\right) \Psi_{\boldsymbol{k}_{\alpha}^{\prime}, \boldsymbol{q}_{\alpha}^{\prime}}^{-*}\left(\boldsymbol{r}_{\alpha}^{\prime}, \boldsymbol{\rho}_{\alpha}^{\prime}\right)}{E-k_{\alpha}^{\prime 2} / 2 \mu_{\alpha}-q_{\alpha}^{\prime 2} / 2 M_{\alpha}+i 0} \\
+\sum_{n_{\alpha}} \int \frac{d \boldsymbol{q}_{\alpha}^{\prime}}{(2 \pi)^{3}} \frac{e^{i \boldsymbol{q}_{\alpha}^{\prime} \cdot \boldsymbol{\rho}_{\alpha}-i \bar{\eta}_{\alpha} / q_{\alpha}^{\prime} \ln \zeta\left(\boldsymbol{q}_{\alpha}^{\prime}, \boldsymbol{\rho}_{\alpha}\right)} \phi_{n_{\alpha}}\left(\boldsymbol{r}_{\alpha}\right) \Phi_{n_{\alpha}, \boldsymbol{q}_{\alpha}^{\prime}\left(\boldsymbol{r}_{\alpha}^{\prime}, \boldsymbol{\rho}_{\alpha}^{\prime}\right)}^{(\alpha)}}{E-E_{n_{\alpha}}-q_{\alpha_{\alpha}^{\prime}}^{\prime 2} / 2 M_{\alpha}+i 0} .
\end{aligned}
$$

Calculating the integrals, we arrive at

$$
\begin{aligned}
G^{+}\left(\boldsymbol{r}_{\alpha}, \boldsymbol{\rho}_{\alpha} ; \boldsymbol{r}_{\alpha}^{\prime}, \boldsymbol{\rho}_{\alpha}^{\prime} ; E+i 0\right) \stackrel{\left(r_{\alpha}, \rho_{\alpha}\right) \in \Omega_{\alpha}}{\longrightarrow} & \frac{(2 \pi i)^{1 / 2}}{(2 \pi)^{3}} \frac{\left(\mu_{\alpha} M_{\alpha}\right)^{3 / 2}}{m^{2}} \frac{\kappa^{3 / 2}}{R^{5 / 2}} \\
& \times \Psi_{\left(\mu_{\alpha} / m \kappa / R\right) \boldsymbol{r}_{\alpha},\left(M_{\alpha} / m \kappa / R\right) \boldsymbol{\rho}_{\alpha}}^{-*}\left(\boldsymbol{r}_{\alpha}^{\prime}, \boldsymbol{\rho}_{\alpha}^{\prime}\right) \psi_{\alpha}\left(\boldsymbol{K}_{\alpha}, \boldsymbol{r}_{\alpha}\right) \exp \left[i \kappa R-i \lambda_{\alpha} \ln (2 \kappa R)-i \sigma_{\alpha}\right] \\
& -\frac{M_{\alpha}}{2 \pi} \sum_{n_{\alpha}} \frac{e^{i q_{n_{\alpha}} \rho_{\alpha}}}{\rho_{\alpha}} e^{-i \bar{\eta}_{\alpha} / q_{n_{\alpha}} \ln \left(2 q_{n_{\alpha}} \rho_{\alpha}\right)} \Phi_{n_{\alpha}, q_{n_{\alpha}}}^{(\alpha) *} \hat{\boldsymbol{\rho}}_{\alpha}\left(\boldsymbol{r}_{\alpha}^{\prime}, \boldsymbol{\rho}_{\alpha}^{\prime}\right) \phi_{n_{\alpha}}\left(\boldsymbol{r}_{\alpha}\right) .
\end{aligned}
$$

If both $\left(r_{\alpha}, \rho_{\alpha}\right)$ and $\left(r_{\alpha}^{\prime}, \rho_{\alpha}^{\prime}\right) \in \Omega_{\alpha}$, we have

$$
\begin{aligned}
G^{+}\left(\boldsymbol{r}_{\alpha}, \boldsymbol{\rho}_{\alpha} ; \boldsymbol{r}_{\alpha}^{\prime}, \boldsymbol{\rho}_{\alpha}^{\prime} ; E+i 0\right) \stackrel{\begin{array}{c}
\left(r_{\alpha}, \rho_{\alpha}\right) \in \Omega_{\alpha} \\
\left(r_{\alpha}^{\prime}, \rho_{\alpha}^{\prime}\right) \in \Omega_{\alpha}
\end{array}}{\longrightarrow} & \frac{(2 \pi i)^{1 / 2}}{(2 \pi)^{3}} \frac{\left(\mu_{\alpha} M_{\alpha}\right)^{3 / 2}}{m^{2}} \frac{\kappa^{3 / 2}}{R^{5 / 2}} \psi_{\alpha}^{*}\left(\boldsymbol{K}_{\alpha}^{\prime}, \boldsymbol{r}_{\alpha}^{\prime}\right) \psi_{\alpha}\left(\boldsymbol{K}_{\alpha}, \boldsymbol{r}_{\alpha}\right) \\
& \times \exp \left[i \kappa R-i \kappa R^{\prime}\left(\sin \varphi_{\alpha} \sin \varphi_{\alpha}^{\prime} \hat{\boldsymbol{r}}_{\alpha} \cdot \hat{\boldsymbol{r}}_{\alpha}^{\prime}+\cos \varphi_{\alpha} \cos \varphi_{\alpha}^{\prime} \cdot \hat{\boldsymbol{\rho}}_{\alpha} \cdot \hat{\boldsymbol{\rho}}_{\alpha}^{\prime}\right)\right] \\
& \times \exp \left[-\frac{i}{\kappa} \sum_{\nu=\beta, \gamma}\left(\frac{m}{\mu_{\nu}}\right)^{1 / 2} \frac{\eta_{\nu}}{\sin \varphi_{\nu}} \ln \frac{2 r_{\nu}}{r_{\nu}^{\prime}\left(1+\hat{\boldsymbol{r}}_{\nu} \cdot \hat{\boldsymbol{r}}_{\nu}^{\prime}\right)}\right] \\
& -\frac{M_{\alpha}}{2 \pi} \sum_{n_{\alpha}} \frac{e^{i q_{n_{\alpha}} \rho_{\alpha}-i q_{n_{\alpha}} \rho_{\alpha}^{\prime} \cdot \hat{\boldsymbol{\rho}}_{\alpha} \cdot \hat{\boldsymbol{\rho}}_{\alpha}^{\prime}}}{\rho_{\alpha}} \exp \left[-\frac{i \bar{\eta}_{\alpha}}{q_{n_{\alpha}}} \ln \frac{2 \rho_{\alpha}}{\rho_{\nu}^{\prime}\left(1+\hat{\boldsymbol{\rho}}_{\alpha} \cdot \hat{\boldsymbol{\rho}}_{\alpha}^{\prime}\right)}\right] \phi_{n_{\alpha}}^{*}\left(\boldsymbol{r}_{\alpha}^{\prime}\right) \phi_{n_{\alpha}}\left(\boldsymbol{r}_{\alpha}\right) .
\end{aligned}
$$

From Eq. (61) one can get an asymptotic Green's function for the case when $\left(r_{\alpha}, \rho_{\alpha}\right) \in \Omega_{0}$ but $\left(r_{\alpha}^{\prime}, \rho_{\alpha}^{\prime}\right) \in \Omega_{\alpha}$. Clearly, in this case, bound states do not contribute. The leadingorder asymptotic terms of the Green's function for threeparticles interacting via short-range potentials were given by [34].

\section{CONCLUSION}

Summarizing, asymptotic behavior of the scattered wave function describing breakup processes in a system of three arbitrary charged particles has been investigated. Leadingorder terms of the scattered wave are given for asymptotic domains where all three particles are widely separated and when any two are close to each other but far from the third particle. The derivations are based on the relationship between the total wave function of a breakup process in a Coulomb three-body system and wave function of the process of scattering of all three particles of the system in the continuum. A consequence of the relationship is that the forms presented in the present work are free from the phase ambiguities that are a feature of the previously known form. A similar technique is used to obtain asymptotic forms of the 
three-body Coulomb Green's function.

The derived wave functions are suitable for use in calculations of ionization in electron/positron-atom and ion-atom collisions, double-photoionization of helium and similar breakup processes in nuclear physics. For instance, the breakup amplitude can be extracted by the direct comparison of the numerically calculated wave function for sufficiently large hyper-radius with its analytic asymptotic forms given in the present work. More sophisticated methods of extraction of breakup amplitudes are being currently investigated.

\section{ACKNOWLEDGMENTS}

The work was supported by the Australian Research Council, U.S. DOE under Grant No. DE-FG03-93ER40773, and NSF under Grant No. PHY-0140343.
[1] M. Baertschy, T.N. Rescigno, and C.W. McCurdy, Phys. Rev. A 64, 022709 (2001).

[2] M.S. Pindzola, D. Mitnik, and F. Robicheaux, Phys. Rev. A 62, 062718 (2000).

[3] K. Bartschat, E.T. Hudson, M.P. Scott, P.G. Burke, and V.M. Burke, J. Phys. B 29, 115 (1996).

[4] I. Bray and A.T. Stelbovics, Phys. Rev. Lett. 70, 746 (1993).

[5] J.L. Friar and G.L. Payne, in Coulomb Interactions in Nuclear and Atomic Few-Body Collisions, edited by F.S. Levin and D. Micha (Plenum, New York, 1996), p. 97.

[6] A. Temkin, Phys. Rev. 126, 130 (1962).

[7] R. Poet, J. Phys. B 11, 3081 (1978).

[8] J. Shertzer and J. Botero, Phys. Rev. A 49, 3673 (1994).

[9] Y.D. Wang and J. Callaway, Phys. Rev. A 50, 2327 (1994).

[10] I. Bray, Phys. Rev. Lett. 89, 273201 (2002).

[11] K. Bartschat, S. Riordan, and G. Ver Steeg, Phys. Rev. A 65, 060701 (2002).

[12] I. Bray, D.V. Fursa, A.S. Kheifets, and A.T. Stelbovics, J. Phys. B 35, R117 (2002).

[13] M.R. Rudge and M.J. Seaton, Proc. R. Soc. London, Ser. A 283, 262 (1965).

[14] M.R.H. Rudge, Rev. Mod. Phys. 40, 564 (1968).

[15] R.K. Peterkop, Theory of Ionization of Atoms by Electron Impact (Colorado Associated University Press, Boulder, 1977).

[16] S. Jones and A.T. Stelbovics, Phys. Rev. Lett. 84, 1878 (2000).

[17] R.K. Peterkop, Izv. Akad. Nauk Latv. SSR, Ser. Fiz. Tekh. 9, 79 (1960).

[18] R.K. Peterkop, Zh. Eksp. Teor. Fiz. 43, 616 (1962) [Sov. Phys. JETP 14, 1377 (1962)].

[19] J. Nuttall, Phys. Rev. Lett. 19, 473 (1967).
[20] M. Lieber, L. Rosenberg, and L. Spruch, Phys. Rev. D 5, 1330 (1972).

[21] A. Kievsky, M. Viviani, and S. Rosati, Phys. Rev. C 64, 024002 (2001).

[22] L. Rosenberg, Phys. Rev. D 8, 1833 (1973).

[23] A.S. Kadyrov, A.M. Mukhamedzhanov, and A.T. Stelbovics, Phys. Rev. A 67, 024702 (2003).

[24] R.G. Newton, Scattering Theory of Waves and Particles, 2nd ed.. Texts and Monographs in Physics (Springer-Verlag, Berlin, 1982).

[25] L.D. Faddeev and S.P. Merkuriev, Quantum Scattering Theory for Several Particle Systems (Kluwer Academic Publishers, Dordrecht, 1993).

[26] E.O. Alt and A.M. Mukhamedzhanov, J. Phys. B 27, 63 (1994).

[27] E.O. Alt and A.M. Mukhamedzhanov, Phys. Rev. A 47, 2004 (1993).

[28] E.O. Alt and A.M. Mukhamedzhanov, Phys. Rev. A 51, 3852 (1995).

[29] A.M. Mukhamedzhanov, Phys. Rev. A 56, 473 (1997).

[30] P.J. Redmond (unpublished), described in [22].

[31] A.M. Mukhamedzhanov and M. Lieber, Phys. Rev. A 54, 3078 (1996).

[32] L.D. Landau and E.M. Lifshitz, Quantum Mechanics (Nonrelativistic Theory), 3rd ed., Course of Theoretical Physics, Vol. 3 (Pergamon Press, Oxford, 1985).

[33] N. Bleistein and R.A. Handelsman, Asymptotic Expansions of Integrals (Dover Publications, New York, 1986).

[34] R.G. Newton, Ann. Phys. (Leipzig) 74, 324 (1972).

[35] As it will be seen later, the final results do not depend on this complementary constant. 